\title{
Physics Potential of the e-RHIC Based FEL-Nucleus Collider
}

\author{
H. Koru ${ }^{a}$, A. Özcan ${ }^{a}$, S. Sultansoy ${ }^{a, b}$ and Ö. Yavaş ${ }^{c}$ \\ ${ }^{a}$ Physics Dept., Faculty of Science and Arts, \\ Gazi University, 06500 \\ Teknikokullar, Ankara, TURKEY \\ ${ }^{b}$ Institute of Physics, Academy of Sciences, H. Cavid Ave. 33, Baku, \\ AZERBAIJAN \\ ${ }^{c}$ Dept. of Physics Engineering, Faculty of Engineering, Ankara \\ University, 06100 Tandoğan, Ankara, TURKEY
}

\begin{abstract}
Main parameters of the FEL-RHIC collider are estimated. The physics search potential of this machine is illustrated using excitations of the ${ }^{232} \mathrm{Th}$ nucleus as an example. It is shown that, due to the tunability and monochromacity of FEL beam and high statistics, proposed collider will play an important role in the field of "traditional" nuclear physics.
\end{abstract}

Recently, construction of a $10 \mathrm{GeV}$ energy electron linac tangentially to Relativistic Heavy Ion Collider (RHIC, BNL, USA) is proposed [四] in order to investigate the physics of collisions between electrons and heavy ions, and between polarized electrons and polarized protons [2]. Let us cite one paragraph from introduction part of the Ref. [1]:

"There is interest among the synchrotron light community in the construction of a superconducting linac in the energy range from $6 \mathrm{GeV}$ to $10 \mathrm{GeV}$, in order to drive a free electron laser. Except when RHIC is in the (relatively rare) process of cooling down from ambient temperatures, the cryogenic refrigation plant already installed has enough spare capacity to run such a linac. It is therefore natural to place the linac relatively near to RHIC. The synergy is completed by making spare pulses from the linac available for eRHIC collisions." 
In this letter we are arguing that collisions of the Free Electron Laser (FEL) photons with ultra-relativistic nuclei from RHIC will give a unique possibility to investigate nucleus excitations. In estimations below we assume that photon beam is produced by SASE FEL based on $10 \mathrm{GeV}$ TESLA-like linac, taking in mind successful results achieved at the TESLA Test Facility (TTF) Free-Electron Laser at the DESY [3].

A general schematic view of FEL-RHIC collider is given in Fig 1. The photon beam produced by undulator magnet placed at the end of $10 \mathrm{GeV}$ e-linac collides with the nucleus beam from RHIC. Relativistic ions will "see" FEL beam as a "laser" photons with energy $\omega=2 \gamma_{A} \omega_{0}$, where $\gamma_{A}$ is the Lorentz factor of ions and $\omega_{0}$ is the energy of the FEL photons. The FEL-RHIC is a good candidate to become a pioneer of FEL-nucleus colliders, to be followed by FEL-HERA and FEL-LHC [4]. The tunability of $\omega_{0}$ by changing the electron beam energy will give opportunity for resonant production of nucleus excitations. The excited nucleus will turn to the ground state at a distance $\ell=\gamma_{A} \cdot \tau \cdot c$ from collision point, where $\tau$ is the lifetime of the excited state in the nucleus rest frame and $\mathrm{c}$ is the speed of light. Emitted photons will be seen in detector as high energy photons with energies up to $2 \gamma_{A} E_{\gamma}$, where $E_{\gamma}$ is the energy of emitted photons in the rest frame of the nucleus.

The wavelength of the first harmonic of SASE FEL radiation is given by

$$
\lambda=\frac{\lambda_{u}}{2 \gamma_{e}^{2}}\left(1+\frac{k^{2}}{2}\right)
$$

where $\lambda_{u}$ is the period length of an undulator, $\gamma_{e}=E_{e} / m_{e}$ is the Lorentz factor of the electron, $k=e B_{u} \lambda_{u} / 2 \pi m_{e}$ is the undulator parameter, $e=\sqrt{4 \pi \alpha}$ ( $\alpha$ being the fine structure constant) and $B_{u}$ is the peak value of the magnetic field in the undulator. At the TTF FEL with $E_{e}=235 \mathrm{MeV}$ the observed SASE FEL photons has the wavelength $109 \mathrm{~nm}$, which corresponds to $\omega_{0}=11 \mathrm{eV}$. According to Eq. (1), $10 \mathrm{GeV}$ linac based FEL will produce photons with the wavelength $0.06 \mathrm{~nm}$, which corresponds to $\omega_{0}=20 \mathrm{keV}$. On the other hand, the wavelength ot the FEL photons is constrained by $\lambda>4 \pi \varepsilon_{t}$, where $\varepsilon_{t}=\varepsilon_{N} / \gamma_{e}$ and $\varepsilon_{N}$ is normalized emittance of the electron beam. For e-linac based FEL's, $\varepsilon_{N}$ value is usually around $10^{-6} \mathrm{~m} \cdot \mathrm{rad}$, hence $\lambda>0.6 \mathrm{~nm}$ for $E_{e}=10 \mathrm{GeV}$. In order to achieve 
shorter wavelenghts one should either use "cooled" electron beam with smaller emittance or deal with higher FEL harmonics. The last case leads to decrease of the luminosity. For example, the brighteness of the third harmonic (which has 3 times shorter wavelength) is approximately $1 \%$ of the first harmonic one [5]. Combining both methods, the FEL photon energy of $20 \mathrm{keV}$ and even more can be achieved at FEL-RHIC Collider.

Parameters of ${ }^{232} \mathrm{Th}$ beam are presented in Table I, where we use the parameters of gold nucleus for linac-ring option of e-RHIC as reference point [1]. Parameters of SASE FEL beam produced by a $10 \mathrm{GeV}$ TESLA-like linac [6] are given in Table II. Luminosity of the FEL-nucleus collisions is given by

$$
L=\frac{n_{\gamma} n_{A}}{4 \pi \sigma_{x}^{\text {eff }} \sigma_{y}^{\text {eff }}} n_{b} f_{\text {rep }}
$$

where $n_{\gamma}$ and $n_{A}$ are the number of photons and nuclei in the FEL and nucleus bunches, respectively, $n_{b}$ is the number of electron bunches per train, $f_{\text {rep }}$ is TESLA repetition rate, $\sigma_{x}^{e f f}$ and $\sigma_{y}^{e f f}$ are the values which are obtained by choosing the bigger ones of the corresponding horizontal and vertical sizes of the FEL and the nucleus beams (for round beams $\sigma_{x}=\sigma_{y}$ ). Using parameters given in Tables I and II we obtain $L=2 \cdot 10^{30} \mathrm{~cm}^{-2} \mathrm{~s}^{-1}$ with $n_{\gamma}=10^{13}$.

Today, more than hundred excitations of the ${ }^{232} \mathrm{Th}$ nucleus are founded in different experiments [7] and only seven of them are observed in $\left(\gamma, \gamma^{\prime}\right)$ reactions. The reasons are following [8]: low statistics, limited energy resolution, neutron induced background from electron setup material collisions etc. In Table III we present main characteristics of ${ }^{232} \mathrm{Th}$ excitations with low spin $(J \leq 2)$.

The cross section for the resonant photon scattering is given by the well-known BreitWigner formula

$$
\sigma\left(\gamma, \gamma^{\prime}\right)=\frac{\pi}{E^{2}} \cdot \frac{2 J_{e x c}+1}{2\left(2 J_{0}+1\right)} \cdot \frac{B_{\text {in }} B_{\text {out }} \Gamma^{2}}{\left(E-E_{R}\right)^{2}+\Gamma^{2} / 4}
$$

where $E$ is the c.m. energy of the incoming photon (in our case it is very close to that in the rest frame of the nucleus), $J_{e x c}$ and $J_{0}$ are spins of the excited and ground states of the 
nucleus, $B_{\text {in }}$ and $B_{\text {out }}$ are the branching fractions of the excited nucleus into the entrance and exit channels, respectively, $E_{R}$ is the energy at the resonance and $\Gamma$ is the total width of the excited nucleus. Corresponding resonant cross sections are given in Table III.

The energy of FEL photons needed for excitation of corresponding ${ }^{232} \mathrm{Th}$ level can be expressed as:

$$
\omega_{0}=\frac{E_{e x c}}{2 \gamma_{T h}}
$$

where $E_{\text {exc }}$ is the energy of corresponding excited level, $\gamma_{T h}$ is the Lorentz factor of the ${ }^{232} \mathrm{Th}$ nucleus. Corresponding values for needed FEL photon energies are given in Table IV. Taking into account the energy spread of the FEL and the nucleus beam $\left(\Delta E_{\gamma} / E_{\gamma}=\right.$ $\Delta \omega / \omega_{0}=10^{-4}$ and $\left.\Delta E_{A} / E_{A}=10^{-4}\right)$, the approximate value of averaged cross section has been found to be

$$
\sigma_{a v} \approx \sigma_{r e s} \frac{\Gamma}{\Delta E_{\gamma}}
$$

where $\Delta E_{\gamma} \approx 10^{-4} E_{\text {exc }}$. Corresponding values for averaged cross sections are presented in the third column of Table IV. Multiplying these values with the luminosity of FEL-RHIC colliders $\left(2 \cdot 10^{30} \mathrm{~cm}^{-2} \mathrm{~s}^{-1}\right)$, we obtain number of events per second which are given in the last column of Table IV. As seen typical numbers of events are of the order of millions per minute, whereas "traditional" experiments deals with hundreds events.

FEL-RHIC colliders will give opportunity to "measure" unknown decay width using known ones. Indeed, decay width can be estimated using following relation

$$
\Gamma_{(1)} \cong \frac{E_{\gamma}^{(1)}}{E_{\gamma}^{(2)}} \cdot \frac{N_{(1)}}{N_{(2)}} \cdot \frac{\sigma_{\text {res }}^{(2)}}{\sigma_{\text {res }}^{(1)}} \cdot \Gamma_{(2)}
$$

where index 1 (2) corresponds to level with unknown (known) decay width. Let us use the $49.368 \mathrm{keV}$ level as known one and 100 events per second as observation limit. In this case we will be able to "measure" decay width of the 714.25 (1078.7) keV level at FEL -Th RHIC collider, if it exceeds $2.5 \cdot 10^{-7}\left(2.6 \cdot 10^{-6}\right) \mathrm{eV}$.

We had mentioned that the excited nucleus will turn to the ground state at a distance $\ell=\gamma_{A} \cdot \tau \cdot c$ from collision point and the emitted photons will be seen in detector as high 
energy photons with energies up to $2 \gamma_{A} E_{\gamma}$. As an example, $\ell$ is equal to $0.46\left(8.2 \cdot 10^{-4}\right)$ $m$ for the 49.369 (2296) $\mathrm{keV}$ excitation of ${ }^{232} \mathrm{Th}$ nucleus at RHIC. Therefore, the detector could be placed close to the collision region. The photons with 49.369 (2296) keV emitted in the rest frame of the nucleus will be seen in the detector as high-energy photons with energies up to 10.1 (470) MeV.

In fixed target experiments the spin of excited nucleus can be determined using angular distribution of the emitted photons. In our case, this angular distribution will be transferred to the energy distribution in laboratory frame. For spin 1 and 2 cases, angular distributions in the rest frame are given by 8

$$
\begin{gathered}
W(\theta)=\frac{3}{4}\left(1+\cos ^{2} \theta\right) \\
W(\theta)=\frac{5}{4}\left(1-3 \cos ^{2} \theta+4 \cos ^{4} \theta\right)
\end{gathered}
$$

respectively. In laboratory system, these distributions will be seen by detector as energy distributions (for $\gamma>>1$ )

$$
\begin{gathered}
W(x)=\frac{3}{4}\left(x^{2}-2 x+2\right) \\
W(x)=\frac{5}{4}\left(4 x^{4}-16 x^{3}+21 x^{2}-10 x+2\right)
\end{gathered}
$$

where $x=E_{\gamma} / \gamma_{A} \omega$. Here, $x$ varies from 0 to $2\left(x=0\right.$ corresponds to $\theta=180^{0}$ and $x=2$ corresponds to $\theta=0^{0}$ ). Fig. 2 shows the $x$ dependence of normalized energy distributions. Taking into account the high statistics, provided by proposed experiment, it is obvious that different spin values can be easily identified.

In the nucleus rest frame, parity $\pi$ of spin 1 dipole excitations in a nucleus with $0^{+}$ground state can be determined [9] using linearly polarized FEL beam by measuring

$$
\begin{aligned}
\Sigma & =\frac{W\left(\theta=90^{0}, \varphi=0^{0}\right)-W\left(\theta=90^{0}, \varphi=90^{0}\right)}{W\left(\theta=90^{0}, \varphi=0^{0}\right)+W\left(\theta=90^{0}, \varphi=90^{0}\right)} \\
& =\pi_{1}=\left\{\begin{array}{l}
+1, \text { for } J^{\pi}=1^{+}, \\
-1, \text { for } J^{\pi}=1^{-} .
\end{array}\right.
\end{aligned}
$$


In our case, $\theta=90^{\circ}$ corresponds to photons with $E_{\gamma}=\gamma_{A} \omega$, which are emitted at $\eta_{\max }=$ $1 / \gamma_{A}$, where $\eta$ is the angle between emitted photon and initial nucleus beam direction. Azimuthal angle $\varphi$ with respect to the (horizontal) polarization plane of the $\gamma$ beam is unchanged when transferred to the laboratory frame. If the detector is placed at a distance $100 \mathrm{~m}$ from the interaction point, the spot size of emitted photons will have a radius about $1 \mathrm{~m}$. Therefore, the measurement of parity can be made easily.

Finally, let us compare proposed experiment with existing ones. As mentioned in Ref. [8]:

"There are several methods to produce photons for low energy photon scattering experiments ... An ideal photon source for such experiments should have the following characteristics:

- High spectral intensity $I=N_{\gamma} / \mathrm{eV} \cdot s$ (number of photons per energy bin and second),

- Good monochromaticity $\Delta E_{\gamma} / E_{\gamma}$

- Tunable in a broad energy range,

- High degree of linear polarization $\left(P_{\gamma} \approx 100 \%\right)$.

Up to now there are no such ideal sources available fulfilling all these requirements in every respect. Therefore, diverse photon sources have been applied in low energy photon scattering depending on the special experimental requirements and aims intended in the investigations ..."

FEL-RHIC collider fulfills all these requirements! This statement is illustrated by Table $\mathrm{V}$, where characteristics of the different photon sources are presented. Moreover, since the accelerated nuclei are fully ionized, the background which is induced by low-shell electrons in the case of investigation of excitations of heavy nuclei using traditional methods will be eliminated.

In conclusion, we hope that the huge number of events provided by FEL-RHIC collider and pure experimental environment will give opportunity to investigate the most of known excitations of ${ }^{232} \mathrm{Th}$ nucleus ( $\sim 100$ levels), which are observed by different experiments, in $\left(\gamma, \gamma^{\prime}\right)$ reactions, too, as well as to observe a lot of additional levels. Similar analysis can be 
easily performed for other nuclei. In general FEL-RHIC collider will essentially enlarge the role of $\left(\gamma, \gamma^{\prime}\right)$ reactions in nuclear physics research.

We are grateful to A.K. Çiftçi and Y.İslamzade for useful discussions. This work is partially supported by Turkish State Planning Organization under the Grant No 2002 K 120250. 


\section{REFERENCES}

[1] I. Ben-Zvi, J. Kewich, J. Murphy and S. Peggs, Nucl. Instrum. Meth. A463 (2001) 94.

[2] Workshop on eA and polarized ep Collider Physics with eRHIC at BNL, http://quark.phy.bnl.gov/ raju/yale_eRHIC.htm, New Haven, 2000.

[3] J. Andruszkow et al., Phys. Rev. Lett. 85 (2000) 3825.

[4] H. Aktaş et al., Nucl. Instrum. Meth. A428 (1999) 271.

[5] J.Arthur et al., SLAC-R-593 (2002).

[6] R. Brinkmann et al. (Eds), Conceptual desing of a $500 \mathrm{GeV} e^{+} e^{-}$linear collider with integrated X-ray laser facility, DESY 1997-048, ECFA 1997-182.

[7] Nuclear Data Sheets 63 (1991) 156.

[8] U. Kneissl, H.H. Pitz and A. Zilges, Progr. Part. Nucl. Phys. 37 (1996) 349.

[9] N. Pietralla et al., Phys. Rev. Lett. 88 (2002) 012502. 


\section{TABLES}

TABLE I. Parameters of ${ }^{232} \mathrm{Th}$ beam

\begin{tabular}{|l|rr|}
\hline \hline Lorentz factor & & 104 \\
\hline Normalized emittance $(\pi \mu m)$ & 6 \\
\hline Bunch population $\left(10^{9}\right)$ & 1.7 \\
\hline Ring circumference $(m)$ & & 3833 \\
\hline Bunches per ring $(k)$ & & 180 \\
\hline Bunch spacing $(n s)$ & & 71 \\
\hline RMS beam size at the IP $(\mu m)$ & & 60 \\
\hline \hline
\end{tabular}

TABLE II. Parameters of a $10 \mathrm{GeV}$ TESLA -like FEL beam

\begin{tabular}{|c|c|}
\hline Linac repetition rate $(H z)$ & 5 \\
\hline Number of bunches per train $(k)$ & 11000 \\
\hline Photon energy range $(\mathrm{keV})$ & $0.1 \div 20$ \\
\hline Number of photons per bunch $\left(10^{12}\right)$ & $1 \div 100$ \\
\hline Photon beam divergence $(\mu \mathrm{rad})$ & 1 \\
\hline Photon beam diameter $(\mu m)$ & 20 \\
\hline
\end{tabular}


TABLE III. Main characteristics of some of the ${ }^{232} \mathrm{Th}$ nucleus excitations

\begin{tabular}{|c|c|c|c|c|c|}
\hline$E_{\text {exc }}(k e V)$ & $J^{\pi}$ & $\left(\gamma, \gamma^{\prime}\right)$ & $T_{1 / 2}(s)$ & $\Gamma(e V)$ & $\sigma_{\text {res }}\left(\mathrm{cm}^{2}\right)$ \\
\hline 49.369 & $2^{+}$ & + & $345 \cdot 10^{-12}$ & $1.91 \cdot 10^{-6}$ & $5.02 \cdot 10^{-18}$ \\
\hline 714.25 & $1^{-}$ & + & - & - & $1.43 \cdot 10^{-20}$ \\
\hline 774.1 & $2^{+}$ & - & $5.8 \cdot 10^{-12}$ & $1.13 \cdot 10^{-4}$ & $2.03 \cdot 10^{-20}$ \\
\hline 785,3 & $2^{+}$ & - & $2.7 \cdot 10^{-12}$ & $2.43 \cdot 10^{-4}$ & $1.97 \cdot 10^{-20}$ \\
\hline 1053.6 & $2^{+}$ & - & - & - & $1.09 \cdot 10^{-20}$ \\
\hline 1072.9 & $2^{+}$ & - & - & - & $1.06 \cdot 10^{-20}$ \\
\hline 1077.5 & $1^{-}$ & - & - & - & $6.30 \cdot 10^{-21}$ \\
\hline 1078.7 & $0^{+}$ & + & - & - & $2.10 \cdot 10^{-21}$ \\
\hline 1121.8 & $2^{+}$ & - & - & - & $9.69 \cdot 10^{-21}$ \\
\hline 1387.2 & $2^{+}$ & - & $1.4 \cdot 10^{-12}$ & $4.70 \cdot 10^{-4}$ & $6.34 \cdot 10^{-21}$ \\
\hline 1489.3 & $\left(1,2^{+}\right)$ & - & - & - & $(3.30,5.50) \cdot 10^{-21}$ \\
\hline 1554.2 & $2^{+}$ & - & $2.95 \cdot 10^{-12}$ & $2.23 \cdot 10^{-4}$ & $5.05 \cdot 10^{-21}$ \\
\hline 1561.5 & $\left(1,2^{+}\right)$ & - & - & - & $(3.00,5.00) \cdot 10^{-21}$ \\
\hline 1573.0 & $\left(1,2^{+}\right)$ & - & - & - & $(2.96,4.93) \cdot 10^{-21}$ \\
\hline 1578.5 & $2^{+}$ & - & - & - & $4.90 \cdot 10^{-21}$ \\
\hline 1738.1 & $\left(1,2^{+}\right)$ & - & - & - & $(2.92,4.04) \cdot 10^{-21}$ \\
\hline 2043 & $1^{+}$ & + & $8.64 \cdot 10^{-15}$ & 0.076 & $1.75 \cdot 10^{-21}$ \\
\hline 2248 & $1^{+}$ & + & $1.8 \cdot 10^{-14}$ & 0.037 & $1.45 \cdot 10^{-21}$ \\
\hline 2274 & $1^{+}$ & + & $3.9 \cdot 10^{-14}$ & 0.017 & $1.41 \cdot 10^{-21}$ \\
\hline 2296 & $1^{+}$ & + & $2.64 \cdot 10^{-14}$ & 0.025 & $1.39 \cdot 10^{-21}$ \\
\hline
\end{tabular}


TABLE IV. The ${ }^{232}$ Th excitations at the FEL-RHIC collider

\begin{tabular}{|c|c|c|c|}
\hline$E_{\text {exc }}(k e V)$ & $\omega_{0}(e V)$ & $\sigma_{a v}\left(\mathrm{~cm}^{2}\right)$ & $N_{\text {events }} / \mathrm{s}$ \\
\hline 49.369 & 237 & $1.94 \cdot 10^{-24}$ & $3.88 \cdot 10^{6}$ \\
\hline 714.25 & 3433 & - & - \\
\hline 774.1 & 3721 & $2.96 \cdot 10^{-26}$ & $5.92 \cdot 10^{4}$ \\
\hline 785.3 & 3775 & $6.09 \cdot 10^{-26}$ & $1.22 \cdot 10^{5}$ \\
\hline 1053.6 & 5065 & - & - \\
\hline 1072.9 & 5158 & - & - \\
\hline 1077.5 & 5180 & - & - \\
\hline 1078.7 & 5186 & - & - \\
\hline 1121.8 & 5393 & - & - \\
\hline 1387.2 & 6669 & $2.15 \cdot 10^{-26}$ & $4.30 \cdot 10^{4}$ \\
\hline 1489.3 & 7160 & - & - \\
\hline 1554.2 & 7472 & $7.24 \cdot 10^{-27}$ & $1.45 \cdot 10^{4}$ \\
\hline 1561.5 & 7507 & - & - \\
\hline 1573.0 & 7562 & - & - \\
\hline 1578.5 & 7588 & - & - \\
\hline 1738.1 & 8356 & - & - \\
\hline 2043 & 9822 & $6.51 \cdot 10^{-25}$ & $1.30 \cdot 10^{6}$ \\
\hline 2248 & 10807 & $2.39 \cdot 10^{-25}$ & $4.78 \cdot 10^{5}$ \\
\hline 2274 & 10932 & $1.05 \cdot 10^{-25}$ & $2.10 \cdot 10^{5}$ \\
\hline 2296 & 11038 & $1.51 \cdot 10^{-25}$ & $3.02 \cdot 10^{5}$ \\
\hline
\end{tabular}


TABLE V. Characteristics of the different photon sources (for details and corresponding references see [8]).

\begin{tabular}{|l|c|c|c|c|c|}
\hline \hline Photon Source & $L C P$ & $B S_{p}$ & $B S_{u p}+C P$ & $B S_{u p}$ & $F E L-R H I C$ \\
\hline Spectral Intensity $(\gamma / \mathrm{s} \cdot \mathrm{eV})$ & 0.15 & 20 & 1000 & 1000 & $10^{16} \cdot M e V / E_{\text {exc }}$ \\
\hline$\Delta \mathrm{E}_{\gamma} / \mathrm{E}_{\gamma}(\%)$ & 2.7 & cont. & cont. & cont. & 0.01 \\
\hline $\mathrm{P}_{\gamma}(\%)$ & 100 & $10-30$ & $10-20$ & 0 & 100 \\
\hline Target Mass M (g) & 70 & 5 & 5 & $1-2$ & $10^{-10}$ \\
\hline \hline
\end{tabular}




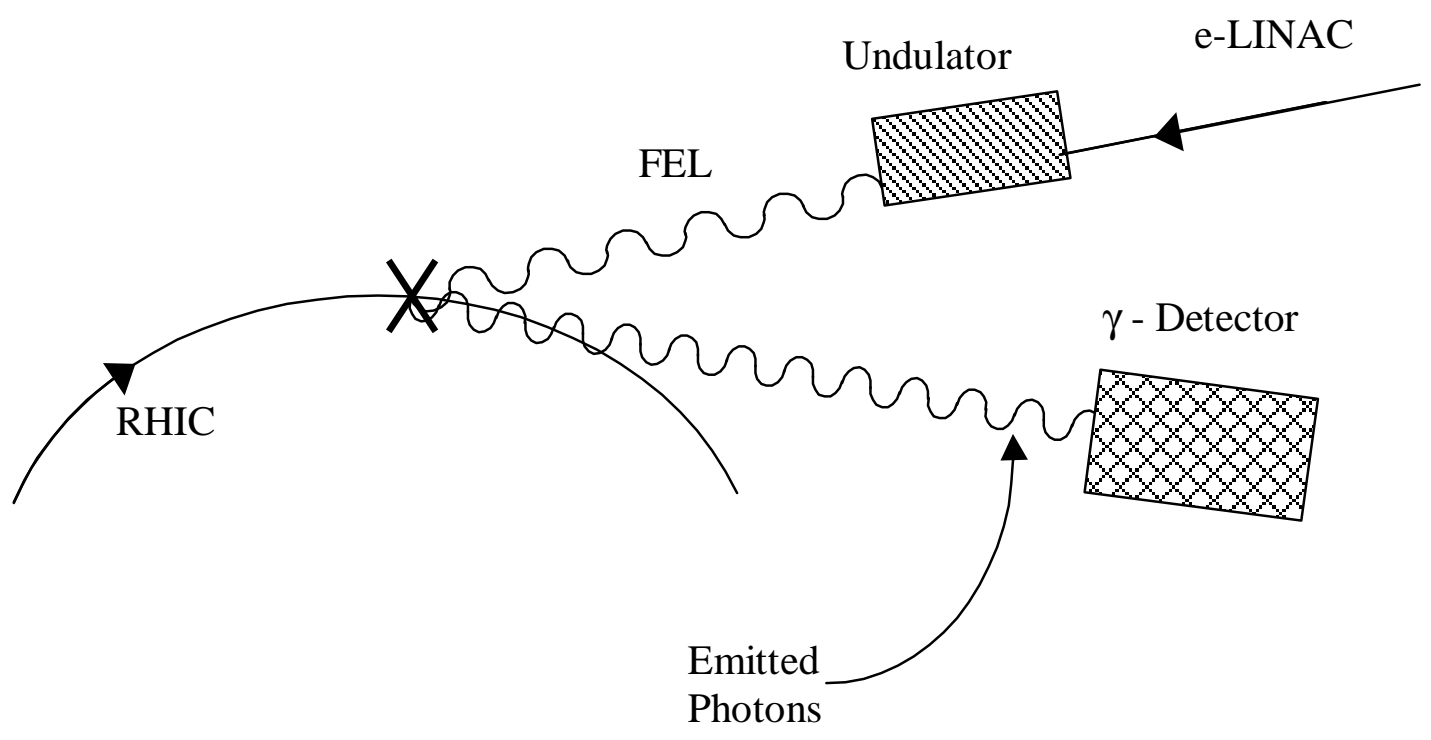

Figure 1. A general schematic view of the FEL- RHIC Collider

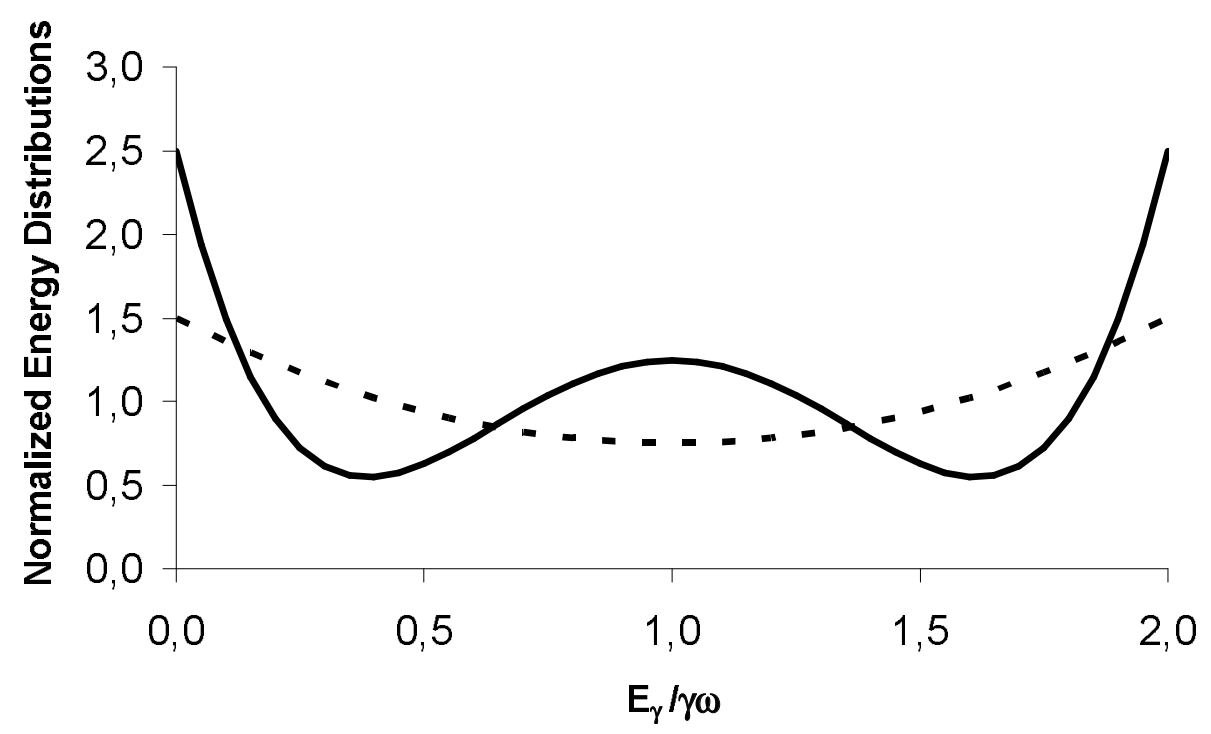

Figure 2. Normalized energy distrubitions of photons emitted by spin 1 (dashed curve) and spin 2 (continous curve) excitations of the ${ }^{232}$ Th nucleus. 